\title{
Study of transient phenomena using NI-USB data acquisition systems in Matlab-Simulink medium on 64 bit operating systems
}

\author{
Titu Niculescu \\ Department of Control Engineering, Computers, Electrical Engineering and Power Engineering University of Petrosani \\ E-mail: niculescu_titu@yahoo.com
}

Copyright $\odot 2015$ Titu Niculescu. This is an open access article distributed under the Creative Commons Attribution License, which permits unrestricted use, distribution, and reproduction in any medium, provided the original work is properly cited.

\begin{abstract}
The paper presents a new and modern method for study the transient phenomena appeared to connect the capacitive charges to an AC power source, using the MATLAB-SIMULINK software package. It is known that NI-USB dataacquisition systems manufactured by National Instruments are not seen by Simulink software package in 64-bit systems. For this is obligatory for use a 32-bit system. From this point of view, the article presents a method by which this disadvantage is eliminated, making possible the data acquisition process in Simulink software package.
\end{abstract}

Keywords: Capacitive Circuit; Data Acquisition; Diagrams; Differential Equation; Electrical Diagram; Matlab- Simulink; NI-USB.

\section{Introduction}

Data-acquisition systems NI-USB type manufactured by National Instruments allows the real-time evaluation of analog measurements in various practical situations. These quantities can be read by Matlab software, but cannot be processed in Simulink because MathWorks Company does not offer support for this software in 64-bit systems. The article presents a method that makes this possible and is studied connecting inductive-capacitive loads to a voltage power source with data acquisitions in Simulink on 64-bit systems. We can consider for this, a RLC series circuit with concentrated parameters, which will be connected to an AC power source.

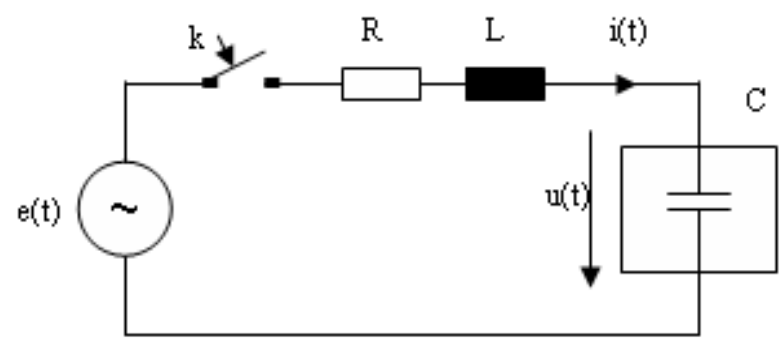

Fig. 1: Capacitor Connecting to an AC Voltage Source

We can meet this situation in practice, in case we want to connect to an AC power source an electrical equipment which includes capacitors. In this case can occur overvoltages or over currents during the transient regime, which can create problems system sizing. The circuit inductivity forms with the capacitors an oscillating circuit (Fig.1). 


\section{Theoretical considerations}

The differential equation for transitory phenomena is presented below, where " $\mathrm{u}(\mathrm{t})$ " is the capacitor voltage.

$$
L C \frac{d^{2} u(t)}{d t^{2}}+R C \frac{d u(t)}{d t}+u(t)=U_{m} \sin (\omega t+\psi)
$$

Where $\Psi$ is the initial phase of voltage when was connected the circuit.

Regime most important and most common is when the circuit resistance is very small, practically the resistance of junction wires. This event is known in the scientific literature as an oscillating regime. If we consider the $\mathrm{R}$ resistance of a circuit very small, the Laplace's method applied to this circuit conducts to the following solution [2]:

$$
\begin{gathered}
u(t)=\frac{U_{m} \sin \left(\omega t+\psi-\phi_{1}\right)}{C \omega \sqrt{R^{2}+\left(\frac{1}{\omega C}-L \omega\right)^{2}}}+\frac{D U_{m} e^{-\delta t}}{2 L C \omega_{e}^{2}} \sin \left(\omega_{e} t-\gamma\right) \\
i=I_{m} \cos \left(\omega t+\psi-\phi_{1}\right)+\frac{D U_{m} e^{-\delta t}}{2 \omega_{e} L} \cdot\left[\cos \left(\omega_{e} t-\gamma\right)-\frac{\delta}{\omega_{e}} \sin \left(\omega_{e} t-\gamma\right)\right]
\end{gathered}
$$

Where $\delta$ is a dumping factor?

$$
\delta=\frac{R}{2 L}
$$

And $\omega 0$ is the resonance angular frequency of the circuit:

$$
\omega_{0}=\frac{1}{\sqrt{L C}}
$$

We also used the following notations:

$$
\begin{aligned}
& \omega_{e}=\sqrt{\omega_{0}^{2}-\delta^{2}} \quad D=\sqrt{A^{2}+B^{2}-2 A B \cos (\beta-\alpha)} \operatorname{tg} \gamma=\frac{A \sin \alpha-B \sin \beta}{A \cos \alpha-B \cos \beta} \\
& \alpha=\phi_{3}+\psi ; \quad \beta=\phi_{2}-\psi \\
& \phi_{1}=\operatorname{arctg} \frac{2 \omega \delta}{\omega_{0}^{2}-\omega^{2}} \quad \phi_{2}=\operatorname{arctg} \frac{\delta}{\omega_{e}-\omega} \quad \phi_{3}=\operatorname{arctg} \frac{\delta}{\omega_{e}+\omega} \\
& A=\frac{1}{\sqrt{\left(1+\frac{\omega}{\omega_{e}}\right)^{2}+\left(\frac{\delta}{\omega_{e}}\right)^{2}}} \quad B=\frac{1}{\sqrt{\left(1-\frac{\omega}{\omega_{e}}\right)^{2}+\left(\frac{\delta}{\omega_{e}}\right)^{2}}} \\
& I_{m}=\frac{U_{m}}{\sqrt{R^{2}+\left(\frac{1}{\omega C}-\omega L\right)^{2}}}
\end{aligned}
$$

\section{Interface circuits necessary for data acquisition}

For experimental evaluation of these parameters a data-acquisition system manufactured by National Instruments, NI USB-6003 type was used. The graphic medium used is MATLAB software, and the electrical diagram is presented in Fig.2. The inductive circuit is connected to the $\mathrm{AC}$ voltage through a capacitor $\mathrm{C}$, and the DAQ input voltage levels are obtained using resistive dividers. The way chosen is the differential measurement.

To eliminate the risk of connecting directly the AC phase voltage to the input of the data-acquisition system, were used symmetrical voltage dividers, analog inputs of the measuring system are connected in parallel with the median divider 
resistors $\mathrm{Ri} 0$ and $\mathrm{RI} 1$. To protect the analog inputs of the data-acquisition system to any voltage surge, the DZ Zener diodes were used.

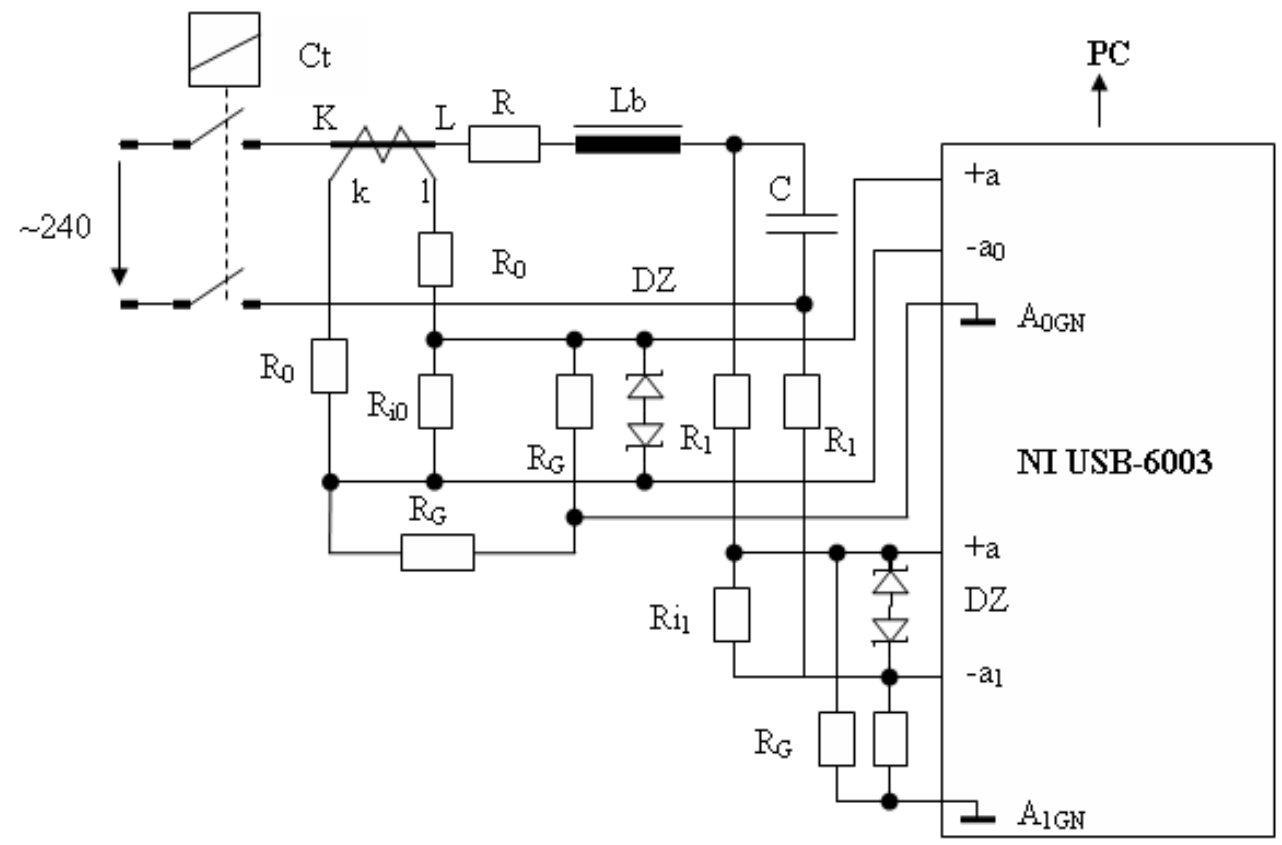

Fig. 2: Electrical Diagram of Measurement System

With measurements were obtained following diagrams of variation of the voltage across the capacitor and the current in the circuit. The diagrams from Fig.3 and Fig.4 were obtained directly in MATLAB, for the following electrical parameters: Fig.3: $\mathrm{U}=220 \mathrm{~V} ; \mathrm{R}=2 \Omega ; \mathrm{L}=90 \mathrm{mH} ; \mathrm{C}=100 \mu \mathrm{F}$;

Fig.4: $\mathrm{U}=220 \mathrm{~V} ; \mathrm{R}=2 \Omega ; \mathrm{L}=2 \mathrm{mH} ; \mathrm{C}=100 \mu \mathrm{F}$;

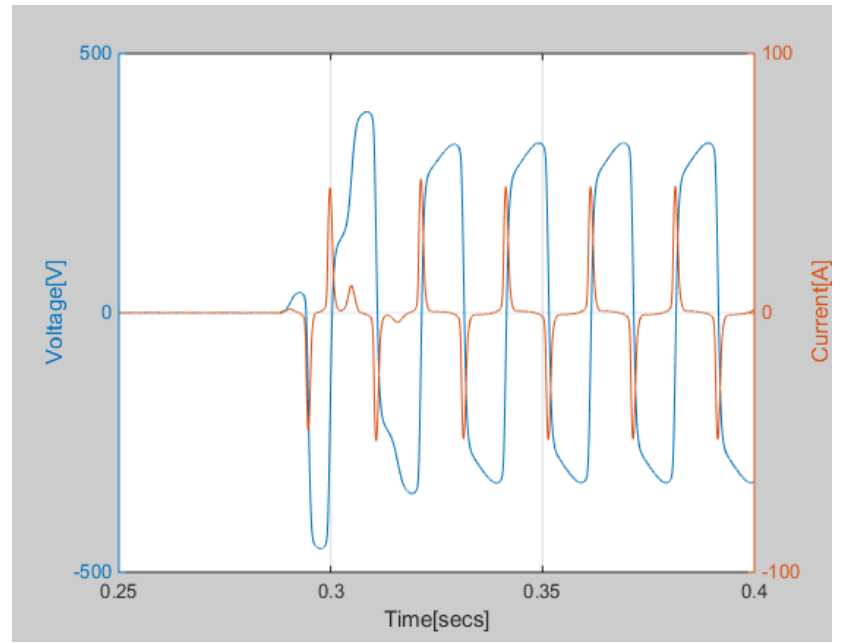

Fig. 3: Variation of Electrical Parameters for $\mathrm{L}=90 \mathrm{mH}$

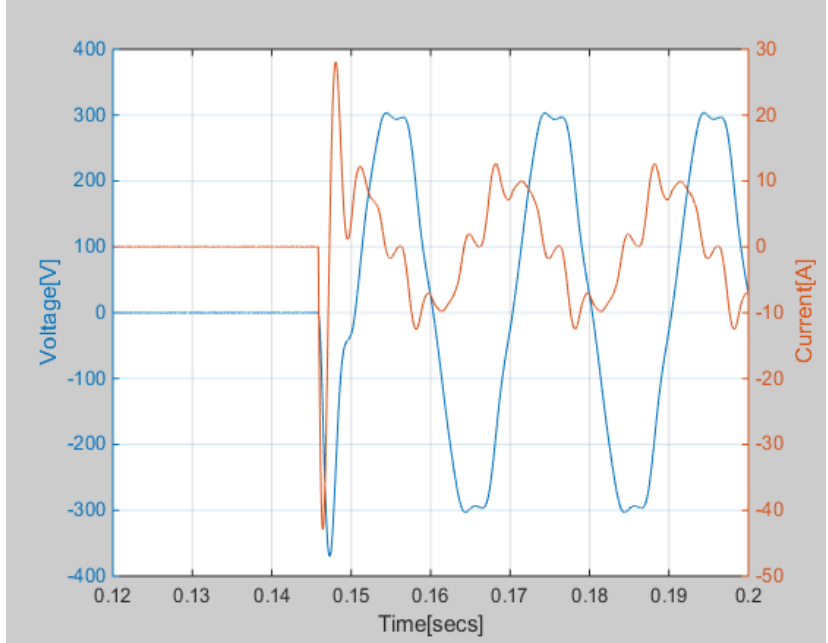

Fig. 4: Variation of Electrical Parameters for $\mathrm{L}=2 \mathrm{mH}$

The most dangerous regime is the oscillatory regime because occurs the highest switching over voltages. For this reason it chosen low value resistance circuit and measurements that were made corresponding to the regime.

\section{Measurement processing in Simulink}

Experimental measurements were made with a data-acquisition system type NI USB-6003 with a rate of $100 \mathrm{kS} / \mathrm{sec}$, and the measurements were processed by 2014b MATALB version, who "sees" only data-acquisition system in Matlab, not in Simulink. Data processing in Simulink involves the following steps:

- It makes the appropriate data acquisition in Matlab (using specific program lines for data-acquisition system type);

- It saves Workspace generated by the measurement (for further processing); 
- The file Data from Workspace opens ;

- The file Time from Workspace opens;

- Undock a command is given to these files;

- It copies the Time column in Data file, so the Time column must be the first.

- It execute Dock Variables command and saves the new Workspace.

At this moment the Data folder from Workspace can be read from a Simulink simulation model. This is shown in Fig.5.

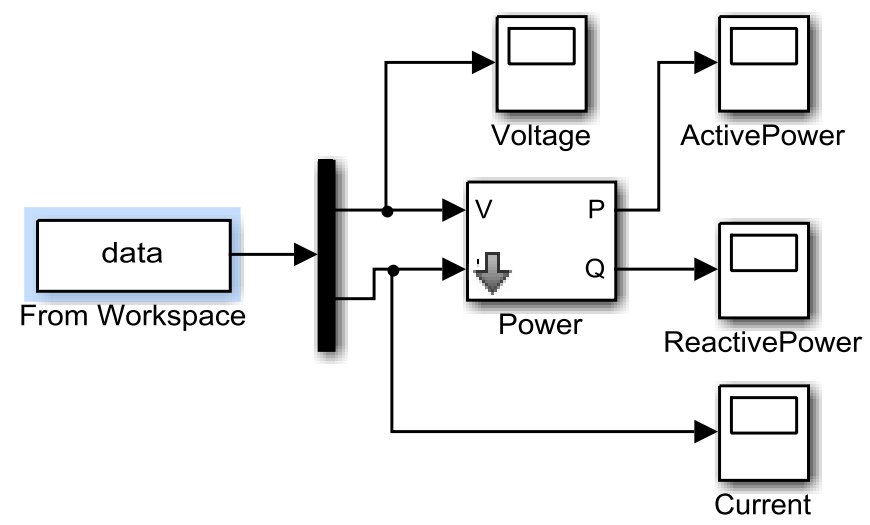

Fig. 5: Simulink Model for Capacitor Connecting

For the inductance coil value of $24 \mathrm{mH}$ were obtained following forms of variation:

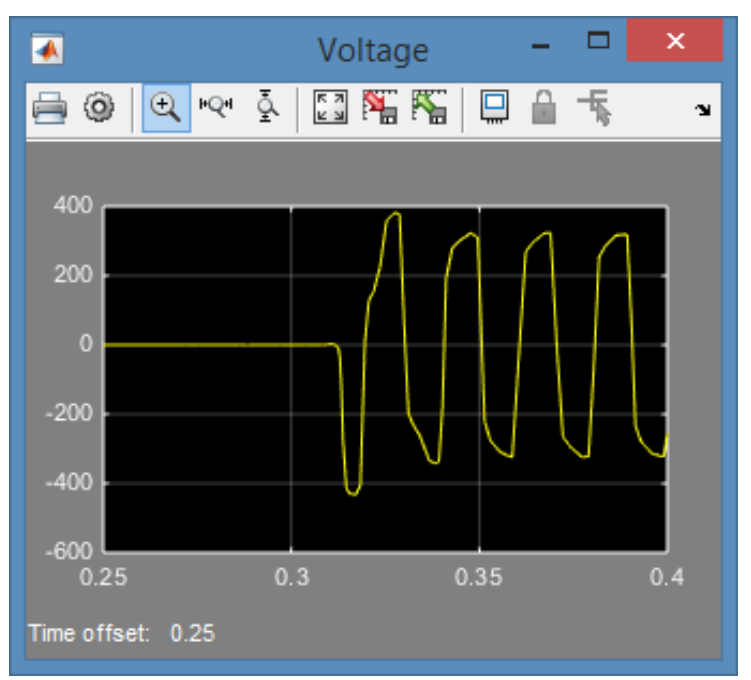

Fig. 6: Capacitor Voltage Variation

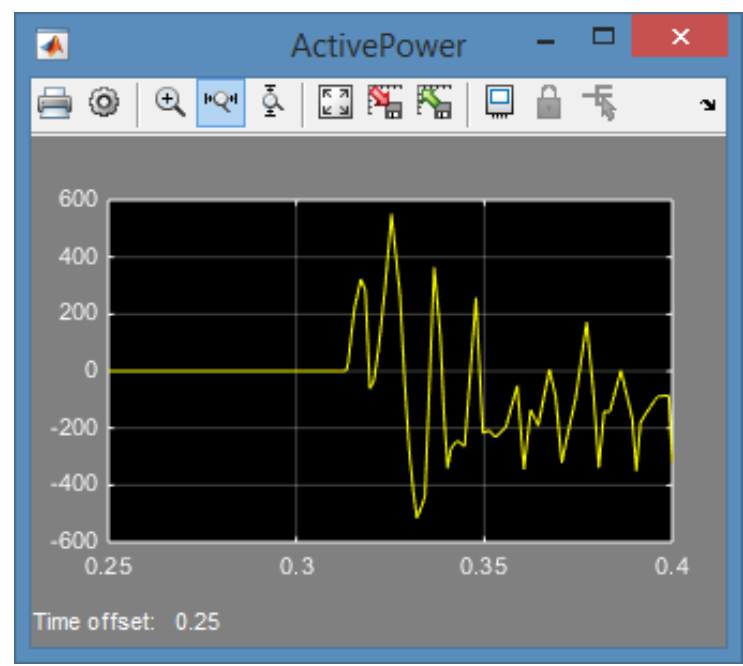

Fig. 8: Active Power Variation

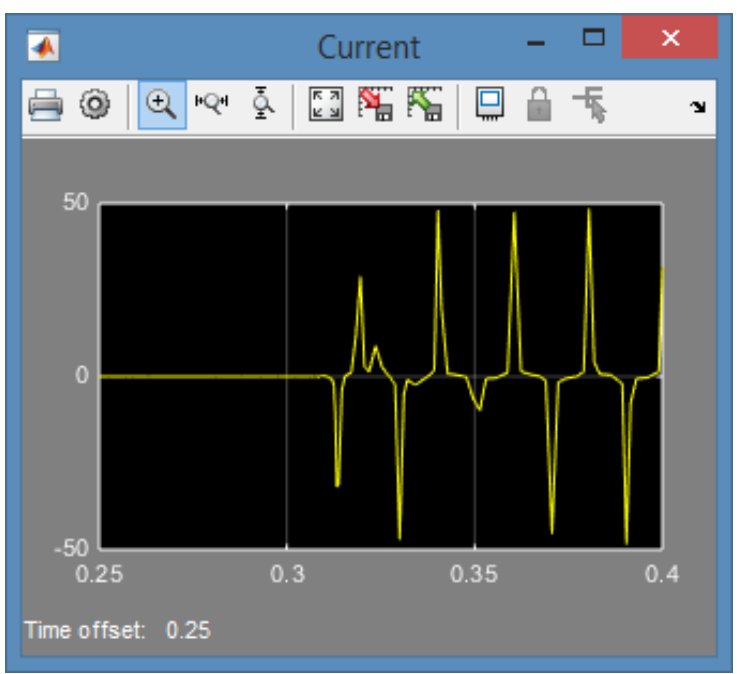

Fig. 7: Capacitor Current Variation

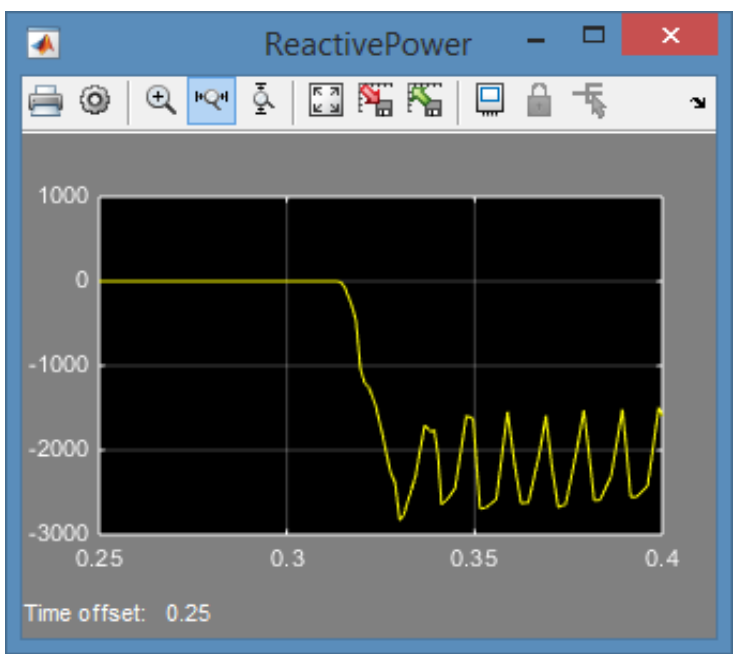

Fig. 9: Reactive Power Variation 
Variation curves of voltage and current, who have the same form as that obtained by direct acquisition in Matlab (Fig.3 and Fig.4) are not sinusoidal due to the presence of reactive elements in the circuit. Because the reactive elements are not ideal, appears on the capacitor a low-value oscillatory active power (approx. 200W). It also appears an oscillating reactive power, but much higher values (up to approx. $3000 \mathrm{VAR}$ ).

If the inductance coil used is a small value (approx. $2 \mathrm{nH}$ ), Simulink model of Fig.5 conduct to obtaining the following diagrams:

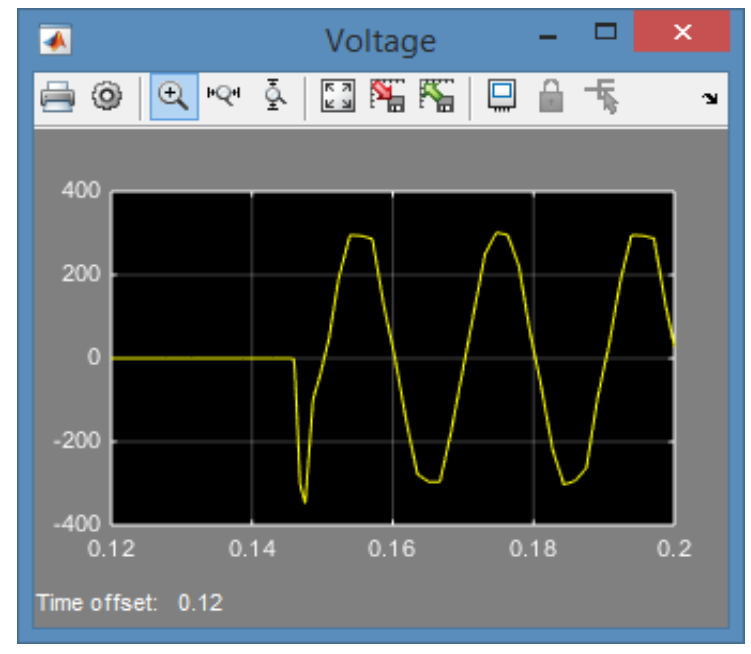

Fig. 10: Capacitor Voltage Variation

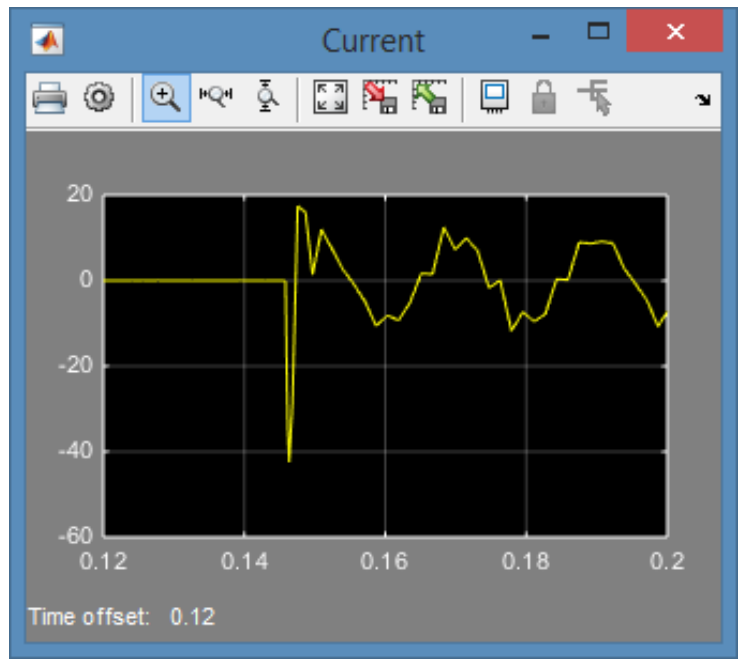

Fig. 11: Capacitor Current Variation

In this case also, the variation forms of voltage and current are identical to those obtained by direct acquisition in Matlab (Fig.4). Due to the small value of inductance, in the current wave, high frequency oscillation occurs, which can lead to undesirable phenomena of electrical resonance. Active power is relatively small because the capacitor is not ideal, and the capacitor reactive power dissipation is significant (aprox. 1500 VAR).

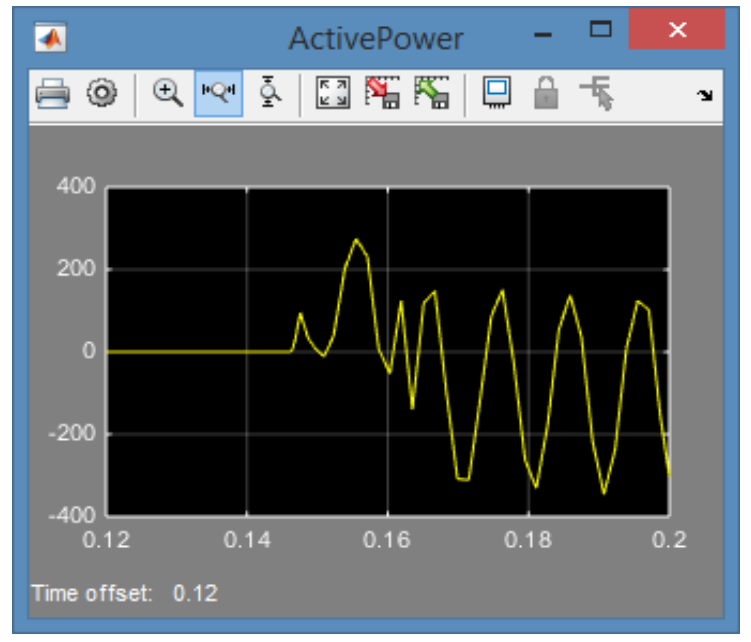

Fig. 12: Active Power Variation

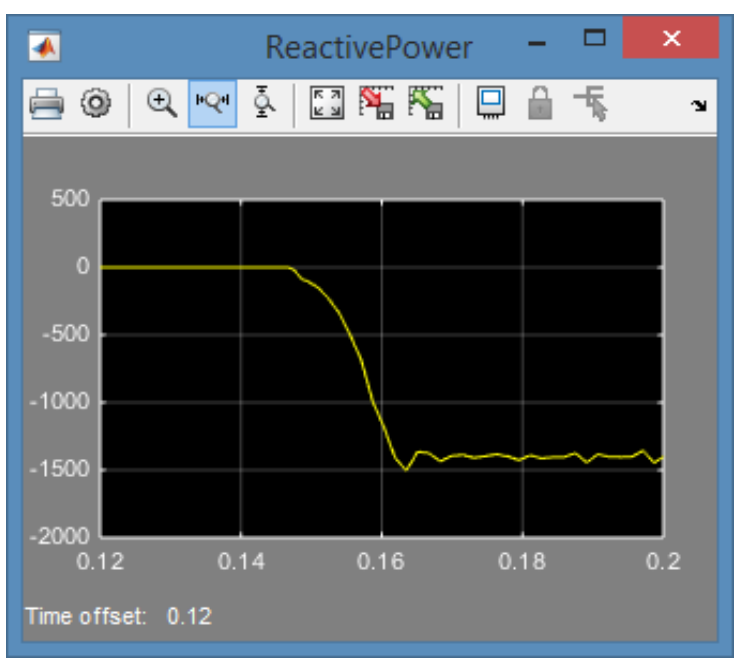

Fig. 13: Reactive Power Variation

\section{Conclusions}

This analysis method allows the study of transient electrical phenomena on 64-bit operating systems. In this case, in Simulink does not appear the DataAcquisitionToolbox package, which is specific to the 32-bit operating systems. We have to mention that the transient regime for connecting the capacitive loads to an AC power source depends on moment connecting given by $\Psi$ angle from relation (1). In this paper we considered a representative case of several measurements that emphasize the higher values of voltage and current occurring immediately after connecting.

The most dangerous regime is the oscillating regime, and this is the most common regime which we can meet in practice. In practice, this could be seen in the alternative power supply for electric motor, which includes starting capacitor. In this case, oscillating overvoltage may occur that need to be taken into account when sizing the systems. The value of these voltages depends on the connecting moment, which is the initial phase of the AC voltage. At the designing and sizing of these circuits must be taken into account overvoltage peaks occurring immediately after 
connection, which can endanger the internal isolation of equipment. Overcurrent peaks in an oscillatory regime can lead to possible switching of protective relays.

It is important that the electric charge on the capacitor which is connected to be initially zero. Otherwise high value over current may occur in the after connecting moment.

\section{References}

[1] $* * *$ Software Matlab 2014b

[2] Hortopan Ghe., "Aparate electrice", E.D.P., Bucure ${ }^{\circ}$ ti, 1980

[3] Horgos, M. et all "Aspects of voltage dips, causes of production and their effect on consumers", Proceedings of the International Conference on ENERGY and ENVIRONME TECHNOLOGIES and EQUIPMENT (EEETE '10) - SPONSOR and ORGANIZER: Facultatea IMST, Universitatea Politehnica, Bucharest, Romania Published by WSEAS Press ISSN: 1790-5095 ISBN: 978-960-474-181-6, pag. 41 - 45.

[4] Nicolae Golovanov, s.a, Consumatori de energie electrica. Materiale. Masurari. Aparate. Instalatii, Editura AGIR, Bucuresti, 2009.

[5] Marin Ghinea, Virgiliu Firteanu, "MATLAB calcul numeric-grafica-aplicatii”, Editura Teora, Bucuresti 2000.

[6] Niculescu, T., Analiza circuitelor electrice prin simulare in spapiul MATLAB, Editura Focus, Petro ${ }^{\circ}$ ani 2006

[7] Niculescu, T. "Technology and Engineering Applications of Simulink", Chapter 2: "Study of Inductive-Capacitive Series Circuits Using the Simulink Software Package” ISBN 978-953-51-0635-7, Hard cover, Publisher: InTech, Published: May 23, 2012 under CC BY 3.0 license, in subject Technology.

[8] NICULESCU T. - Transitory phenomena in capacitive circuits connected to a AC source - Proceedings of the 4 th International Conference on Circuits, Systems, Control, Signals (CSCS'13 and the 1st International Conference on Electronics and Electrical Engineering (ELEL '13), Valencia, Spania August 2013, ISBN: 978-960-474-321-6, pp. 162-166.

[9] Panã, L., Fotãu, I., "Modes of operation study of electric power transformers using the Matlab programme", microCAD 2006, International Scientific Conference 16-17 March, 2006, University of Miskolc, Hungary, pp. 83-88

[10] Panã, L., Marcu, M., and aerban, H., "Modeling and simulation the operational states of electric mining transformers", Annals of the University of Petro ani, Electrical Engineering, vol.8 (XXXV), Universitas Publishing House, Petrosani, Romania , 2006

[11] Simona Halunga-Fratu, Octavian Fratu, Simularea sistemelor de transmisie analogice si digitale folosind mediul MATLAB/SIMULINK, Editura MatrixRom, Bucuresti 2004 